\title{
PRESENÇA DO ACOMPANHANTE DE LIVRE ESCOLHA NO PROCESSO PARTURITIVO: REPERCUSSÕES NA ASSISTÊNCIA OBSTÉTRICA*
}

\author{
Paolla Amorim Malheiros Dulfe ${ }^{1}$, Dalmo Valério Machado de Lima², Valdecyr Herdy Alves², Diego Pereira \\ Rodrigues $^{3}$, Joyce Gonçalves Barcellos ${ }^{4}$, Estefânia de Oliveira Cherem ${ }^{5}$
}

\begin{abstract}
RESUMO: Objetivou-se identificar a prevalência da presença do acompanhante escolhido pela mulher nas fases do processo parturitivo e analisar as associações da presença do acompanhante na assistência obstétrica. Pesquisa quantitativa, retrospectiva de análise documental do banco de dados de um estudo primário, em três maternidades públicas do município de Niterói, Rio de Janeiro, executado em 2014. O Teste de Associação de Qui- quadrado $(\alpha=0,05)$ foi utilizado tendo o Statistical Package for Social Science for Windows como ferramenta. As variáveis manobra de Kristeller, infusão de ocitocina, realização de massagens, técnicas de respiração, oferta de banhos, restrição de movimentação, orientação aos procedimentos realizados, sensação de abandono, isolamento e desrespeito, apresentaram associação significativa com a presença do acompanhante durante esse processo, mostrando- se como fator que influencia no empoderamento feminino, proporciona maior segurança e conforto, reduzindo o abandono e o isolamento. Diante dos seus inúmeros benefícios, a iniciativa deve ser amplamente divulgada e incentivada.
\end{abstract}

DESCRITORES: Parto humanizado; Humanização da assistência; Obstetrícia; Enfermagem obstétrica.

\section{PRESENCE OF A COMPANION OF THE WOMAN'S CHOICE IN THE PROCESS OF PARTURITION: REPERCUSSIONS ON OBSTETRIC CARE}

\begin{abstract}
This study aimed to identify the prevalence of the presence of a companion of the woman's choice in the process of parturition and analyze the associations of the presence of a companion in obstetric care. Quantitative, retrospective and documentary study of a database of a primary study in three public maternity hospitals in the city of Niterói, Rio de Janeiro, conducted in 2014. Chi-square test of association $(a=0.05)$ using SSPS for Windows was performed. The variables Kristeller maneuver, oxytocin infusion, massage, breathing techniques, bathing, restriction of movement, guidance on the procedures performed, feeling of abandonment, isolation and disrespect showed a significant association with the presence of a companion during this process. This indicates that the presence of a companion influences female empowerment, provides greater security and comfort, reducing abandonment and isolation. In view of its many benefits, the initiative should be widely disseminated and encouraged.
\end{abstract}

DESCRIPTORS: Humanized delivery; Humanization of care; Obstetrics; Obstetrical nursing.

\section{PRESENCIA DEL ACOMPAÑANTE DE ELECCIÓN LIBRE EN PROCESO DE PARTO: REPERCUSIONES EN LA ASISTENCIA OBSTÉTRICA}

RESUMEN: Fue objetivo del estudio identificar la prevalencia de la presencia del acompañante escogido por la mujer en las fases del proceso de parto, así como analizar las asociaciones de la presencia del acompañante en la asistencia obstétrica. Investigación cuantitativa, retrospectiva de análisis documental de banco de datos de un estudio primario, en tres maternidades públicas del municipio de Niterói, Rio de Janeiro, hecho en 2014. Se utilizó la Prueba de Asociación de Chi-cuadrado $(a=0,05)$ y el Statistical Package for Social Science for Windows como como herramienta. Las variables maniobra de Kristeller, infusión de ocitocina, realización de masajes, técnicas de respiración, oferta de baños, restricción de movimentación, orientación a los procedimientos realizados, sensación de abandono, aislamiento y falta de respeto presentaron asociación significativa con la presencia del acompañante durante ese proceso, siendo éste un factor que influencía en el empoderamiento femenino, proporcionando más seguridad y bienestar, reduciendo el abandono y el aislamiento. Delante des sus innúmeros beneficios, la iniciativa debe ser largamente divulgada e incentivada.

DESCRIPTORES: Parto humanizado; Humanización de la asistencia; Obstetricia; Enfermería obstétrica.

*Artigo extraído da dissertação intitulada: "Presença do acompanhante de livre escolha durante o processo parturitivo: implicações na assistência instituída à mulher". Universidade Federal Fluminense, 2015.

${ }^{1}$ Enfermeira. Mestranda em Saúde Materno Infantil. Enfermeira Obstetra da Universidade Federal Fluminense. Rio de Janeiro, RJ, Brasil.

${ }^{2}$ Enfermeiro. Doutor em Enfermagem. Professor da Escola de Enfermagem Aurora de Afonso Costa, Universidade Federal Fluminense. Rio de Janeiro, RJ, Brasil.

${ }^{3}$ Enfermeiro. Mestre em Enfermagem. Professor do Centro Universitário Anhanguera de Niterói, Rio de Janeiro, RJ, Brasil.

${ }^{4}$ Enfermeira. Universidade Federal Fluminense. Professora do Centro Educacional de Niterói. Rio de Janeiro, RJ, Brasil.

${ }^{5}$ Enfermeira. Mestre em Saúde Materno Infantil. Enfermeira do Núcleo de Segurança do Paciente do Hospital Sofia Feldman. Minas Gerais, MG, Brasil.

Autor Correspondente:

Recebido: $25 / 08 / 2016$

Diego Pereira Rodrigues

Finalizado: 17/012/2016

Centro Universitário Anhanguera

R. Desembargador Leopoldo Muylaert, 307 - 24350-450 - Niterói, RJ, Brasil

E-mail: diego.pereira.rodrigues@gmail.com 


\section{- INTRODUÇÃO}

As Políticas Públicas que versam sobre a Saúde Materno-Infantil apontam a importância da humanização da assistência em saúde por meio da adoção de práticas baseadas em evidências científicas e que, sobretudo, respeitem os direitos, a individualidade, a integralidade e a autonomia feminina nos diversos cenários de atenção. Tal movimento tornou-se necessário frente à institucionalização do parto, que afastou as mulheres do domicílio em seu contexto íntimo e de amparo familiar, impondoIhes um modelo biomédico de assistência centrado no profissional médico e na instituição hospitalar. No Brasil, a assistência hospitalar ao parto é superior a 95\% desde 1994, atingindo 97,9\% no ano de 2006. Atualmente, as parturientes são assistidas por profissionais habilitados em $95,9 \%$ dos casos, sendo $88 \%$ por médicos e $7,9 \%$ por enfermeiros ${ }^{(1)}$.

Ao chegarem às maternidades, as mulheres frequentemente se deparam com deficiências no acolhimento da equipe de saúde e são submetidas às rotinas hospitalares num ritual baseado na atenção padronizada. Ditam-se normas de comportamento e culturais que acabam por despi-las de sua identidade tornando-as passivas frente ao desconhecimento e às imposições dos profissionais da saúde e das instituições, cujas estruturas físicas e rotinas hospitalares foram planejadas para atender as necessidades desses profissionais, e não das parturientes ${ }^{(2)}$. Ao fim, ainda há de se esperar por partos e nascimentos violentos, sem privacidade, parturientes silenciadas, oprimidas e longe de sua rede de apoio, dela afastada equivocadamente justo no momento em que a presença do acompanhante é imprescindível ${ }^{(3)}$.

Desde 2005, as mulheres tem garantido o direito ao acompanhante de sua livre escolha durante todo trabalho de parto, parto e pós parto imediato, que deve ser acolhido obrigatoriamente pelo Sistema de Saúde. Todavia, apesar de passados quase 11 anos da sanção da Lei $n^{\circ} 11.108$ de 7 de abril de $2005^{(4)}$, o descumprimento do texto legal é facilmente observado nas instituições de saúde. Nos estados brasileiros onde está sendo implementado o Programa Rede Cegonha, estima-se que 62,4\% das mulheres não tiveram assegurado o direito ao acompanhante durante o processo de parturição, conforme previsto na legislação(5).

Assim sendo, é expressivo o quantitativo de mulheres que permaneceram sem acompanhante em qualquer etapa do processo parturitivo(6). Quando investigados os motivos da ausência do acompanhante, constatou-se que $57,3 \%$ dos casos ocorreram em função da proibição das instituições de saúde, contrastando com apenas $14,7 \%$ que desconheciam este direito ${ }^{(5)}$. Por outro lado, entre os pais, predomina o direito de participarem do nascimento de seus filhos, posto que atribuem suas presenças no evento à benevolência da equipe médica, e não à legislação em vigor ${ }^{(7)}$.

Assim, a institucionalização do parto, associada às normas e rotinas hospitalares, ocasionaram o isolamento social das mulheres e o afastamento de seus familiares durante o trabalho de parto e parto, pois são internadas em ambientes coletivos e sem privacidade, além de submetidas às rotinas e à passividade $^{(8)}$.

Considerando a experiência das puérperas acerca da atenção recebida no processo parturitivo, pode-se concluir que as mulheres associam-no a sentimentos de solidão, medo, dor, sofrimento e abandono, uma vez que tiveram seus filhos sozinhas, com assistência limitada ao período expulsivo(3). As evidências científicas vêm mostrando os benefícios do acompanhante durante o trabalho de parto, parto e pós-parto imediato, tais como o conforto, a tranquilidade e a calma propiciados à parturiente pelo suporte recebido. Quanto aos acompanhantes, a simples presença desmistifica temores e sofrimentos, proporcionando sentimentos e emoções únicos ${ }^{(7)}$ atribuídos à experiência positiva, à sensação de satisfação pela vivência e ajuda que fornece à mulher o apoio emocional necessário ${ }^{(2)}$.

Quando em pauta a opinião dos profissionais de saúde que prestam assistência ao parto acerca da presença do acompanhante, destacam-se as mudanças positivas na assistência, o apoio emocional que deixa a parturiente mais satisfeita, segura e tranquila, aspectos considerados positivos no comportamento e participação delas durante o trabalho de parto e parto. No geral, a avaliação demasiadamente positiva é corroborada ainda pelo fato de que os acompanhantes não causaram problemas, mas fizeram diferença na atitude do profissional ou não houve diferença em prestar assistência em presença de acompanhante ${ }^{(2)}$. Entretanto, apesar das evidências, ainda se observa que 
a maioria dos profissionais rejeita a presença do acompanhante no ambiente hospitalar, sentindo-se ameaçados por questionamentos e vendo-o como avaliador de suas práticas assistenciais ${ }^{(9-12)}$.

O presente estudo foi conduzido com os objetivos de identificar a prevalência da presença do acompanhante escolhido pela mulher nas fases do processo parturitivo e analisar as associações da presença do acompanhante na assistência obstétrica. Nesse sentido, traz reflexões que visam a contribuir com a assistência à mulher durante o parto, bem como com toda sua rede de apoio, por meio do entendimento das repercussões benéficas na atenção obstétrica instituída durante o processo de parturição, quando acompanhado por pessoa de sua livre escolha.

\section{MÉTODO}

Este estudo é um recorte da dissertação intitulado: Violência obstétrica no processo do parto e nascimento da região metropolitana II do Estado do Rio de Janeiro: percepção de mulheres/ puérperas $^{(13)}$. Foi desenvolvido um estudo descritivo, com delineamento retrospectivo por meio de análise documental do banco de dados da pesquisa supracitada ${ }^{(13)}$, sendo abordada quantitativamente os dados coletados.

Utilizou-se como participante base do banco de dados da pesquisa original, de onde foram elencadas as três Unidades de Saúde Pública do Município de Niterói, englobando a Rede de Saúde nas esferas municipal, estadual e federal. Foram considerados como critérios de inclusão: mulheres maiores de dezoito (18) anos de idade com permanência maior ou igual a doze (12) horas na unidade de alojamento conjunto; ocorrência de parto vaginal; evolução puerperal fisiológica, respeitando-se os períodos de aleitamento materno e condições psicológicas. Os critérios de exclusão estabelecidos englobaram mulheres em pós-abortamento, pós-parto de natimorto ou neomorto; mulheres com RN internados no complexo neonatal; mulheres que, por quaisquer motivos, tenham estado em setores de cuidados intensivos ou semi-intensivos; e mulheres de outra nacionalidade.

Os dados primários foram coletados no período de janeiro a abril de 2014 e dimensionados a partir do cálculo para variáveis discretas em populações finitas, chegando-se à amostra de aproximadamente 35 mulheres. Entretanto, visando abordar a totalidade dos dados disponíveis nos cenários elencados, obteve-se a amostra equivalente a 42 mulheres em pós-parto, sendo 14 em cada Instituição de saúde.

A presente pesquisa se iniciou em maio de 2014, onde os dados foram acessados, quando se deu o primeiro contato com o banco de dados. As informações obtidas foram selecionadas de acordo com os objetivos desta pesquisa, sendo tabulados por meio do programa Statistical Package for Social Science for Windows (SPSS), versão 17.0.2 para Windows, licença 33430-001 S1702CDL-0209w1, utilizado como ferramenta para a análise estatística.

A partir do banco de dados produzido, utilizou-se a estatística inferencial com o Teste de Associação de Independência por meio do Qui-quadrado, obedecendo-se aos critérios de restrições ${ }^{(14)}$ com o objetivo de associar a presença do acompanhante no processo de parturição com a assistência obstétrica recebida nas maternidades públicas do Município de Niterói, Rio de Janeiro, Brasil. Nas situações em que ele não foi contemplado, utilizou-se o Teste de Fischer. Por convenção, foi estabelecido o nível de significância de $5 \%(\alpha=0,05)$ para determinar os resultados estatisticamente significantes.

Por se tratar de estudo de natureza secundária, não houve necessidade de nova submissão ao Comitê de Ética em Pesquisas ${ }^{(15)}$, posto que o estudo original já havia sido aprovado, pelo Comitê de Ética em Pesquisa da Faculdade de Medicina do Hospital Universitário Antônio Pedro, Universidade Federal Fluminense sob protocolo ${ }^{\circ}$ 375.252/13.

\section{- RESULTADOS}

A distribuição sociodemográfica dos sujeitos demonstrou predominância de mulheres negras, protestantes, que se enquadravam no primeiro grupo de faixa etária, de 18 a 25 anos. Em termos quantitativos 38 (90\%) tinham até 33 anos de idade, com média de 25,6 anos. 
Vinte mulheres $(47,6 \%)$ concluíram o ensino médio e uma delas $(2,4 \%)$ estava cursando o ensino superior. Quanto à situação laboral, apresentaram grande diversificação de ocupações, sendo que $14(33,3 \%)$ eram do lar, seguidas de operadora de caixa e estudante. Vinte e oito mulheres $(66,7 \%)$ não possuíam vínculo empregatício firmado por meio de carteira assinada ou outra fonte de renda. Quando presente, a pensão era responsável pela fonte de renda adicional. Ao todo, apresentaram renda familiar em torno de 1 a 2 salários mínimos $(76,2 \%)$, no valor de $R \$ 724,00$, vigente à época da pesquisa. Vinte e duas entrevistadas (52,4\%) residiam em imóvel próprio, onde coabitavam de 4 a 6 moradores $(59,5 \%)$ distribuídos em cerca de 4 a 5 cômodos $(69 \%)$.

Em relação à condição marital, $11(26,2 \%)$ eram casadas, prevalecendo a união estável. Dentre as entrevistadas, $37(88,1 \%)$ possuíam companheiro atualmente, com tempo médio de relacionamento entre 1 e 3 anos, sendo estes os pais dos bebês recém-nascidos. Quando indagadas sobre o número de filhos vivos, os recém-nascidos apresentaram-se predominantemente como filhos únicos $(42,9 \%)$, com média de dois filhos por mulher.

Com relação aos dados obstétricos, $16(38,1 \%)$ eram primigestas, seguido das secundigestas $(\mathrm{n}=12$; 28,6\%). Desta forma, 21 (50\%) mulheres pariu uma única vez, via vaginal. Anteriormente ao parto atual, somente três mulheres $(7,1 \%)$ foram expostas à cirurgia cesariana.

Quanto ao planejamento da gravidezatual, houve equilíbrio entre os grupos, embora predominassem as gestações não planejadas $(n=24 ; 57,1 \%)$. Apesar disto, oito $(19 \%)$ vivenciaram uma situação anterior de abortamento, independente da natureza.

Nas mulheres avaliadas, as gestações atuais obtiveram desfecho fisiológico dominante entre 39 e 40 semanas de idade gestacional $(n=27 ; 64,3 \%)$ estando compreendidas no termo completo.

Nas instituições estudadas, foi observada a presença do acompanhante durante todo o processo de parturição. Assim sendo, os diversos momentos da internação foram categorizados segundo a Admissão, o Trabalho de Parto e o Parto propriamente dito, conforme consta na Figura 1.

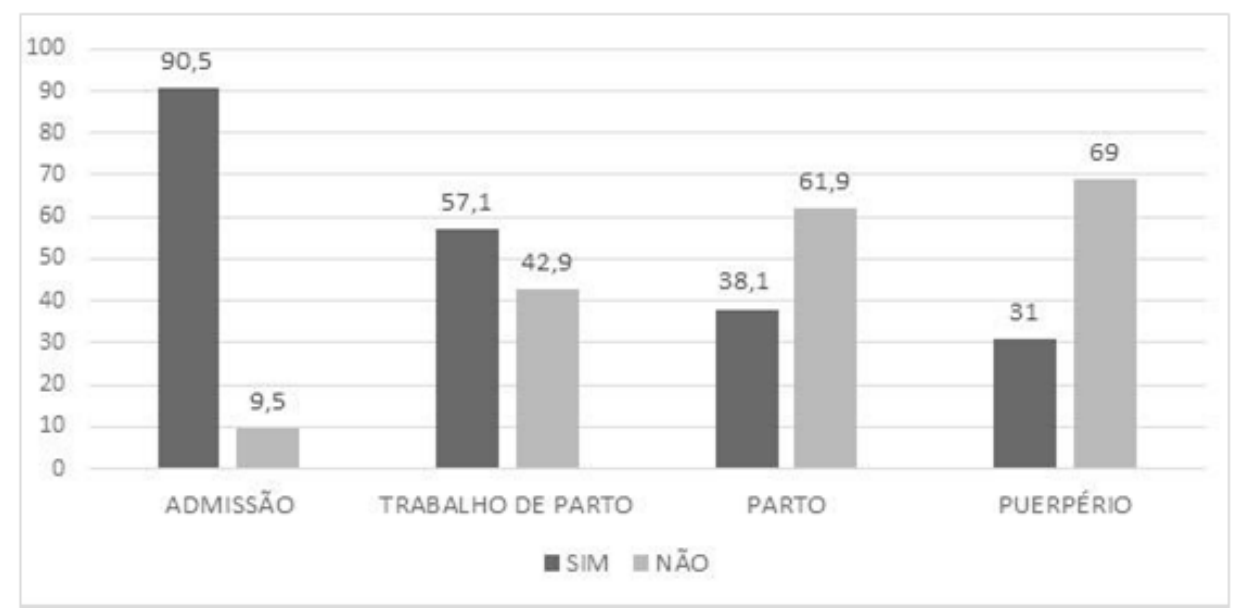

Figura 1 - Presença do acompanhante de livre escolha da mulher durante o processo parturitivo nas maternidades públicas de Niterói. Niterói, RJ, Brasil, 2014 ( $\mathrm{n=42)}$

Durante a admissão obstétrica, 38 (90,5\%) chegaram acompanhadas às instituições de saúde. Contudo, mesmo com a presença maciça dos acompanhantes no momento da internação, $24(57,1 \%)$ tiveram um acompanhante durante o trabalho de parto, sendo que $16(38,1 \%)$ permaneceram acompanhadas no momento do parto, e em quantitativo ainda menor no período puerperal $(n=13 ; 31 \%)$, demonstrando a tendência de limitação do acompanhante no decorrer dos momentos de internação.

Visando a avaliar a influência do acompanhante de livre escolha da mulher na assistência recebida e presenciada pelo acompanhante durante o processo parturitivo, foi realizado o Teste de Associação de Qui-quadrado, apresentado no Quadro 1. 
Quadro 1 - Associação entre a presença do acompanhante e as atividades desempenhadas durante o processo de parturição nas maternidades públicas de Niterói. Niterói, RJ, Brasil, 2014 (N=42)

\begin{tabular}{|c|c|c|}
\hline \multirow[t]{2}{*}{ Procedimentos/Atividades } & \multicolumn{2}{|c|}{$\begin{array}{l}\text { Teste de Associação de Qui-quadrado } \\
\text { p valor }\end{array}$} \\
\hline & Trabalho de Parto & Parto \\
\hline Realização de Massagens & $\mathbf{0 , 0 3}$ & $0,032 *$ \\
\hline Oferta de Banho & $\mathbf{0}$ & $\mathbf{0 , 0 1 1}$ \\
\hline Realização de Técnica de Respiração & $0,055^{*}$ & 0,016* \\
\hline Uso de Bola Suíça & $0,208^{*}$ & $0,690^{*}$ \\
\hline Restrição de Deambulação e Mudança de Posição & 0,003 & $\mathbf{0 , 0 0 7 *}$ \\
\hline Restrição de Alimentos e Líquidos & $0,060^{*}$ & $0,061^{*}$ \\
\hline Administração de Ocitocina & $\mathbf{0 , 0 0 9}$ & 0,078 \\
\hline Realização de Analgesia Epidural ou Raquidiana & $1,000^{*}$ & $0,547^{*}$ \\
\hline Realização de Amniotomia & $0,731^{*}$ & $0,720 *$ \\
\hline Realização de Episiotomia & + & $0,465^{*}$ \\
\hline Realização de Manobra de Valsalva & $1,000^{*}$ & $1,000 *$ \\
\hline Realização de Manobra de Kristeller & + & 0,016 \\
\hline Orientação Quanto aos Procedimentos Realizados & 0,049 & 0,015* \\
\hline Orientação Quanto aos Motivos da Infusão de Ocitocina & $0,040 *$ & $0,048 *$ \\
\hline Informação Quanto ao Exame de Toque Vaginal & $0,685^{*}$ & $1,000^{*}$ \\
\hline Informações sobre o Trabalho de Parto e Parto & $0,679 *$ & $1,000^{*}$ \\
\hline Identificação dos Profissionais de Saúde & $\mathbf{0}$ & $\mathbf{0 , 0 0 7}$ \\
\hline Profissionais se Dirigiam Pelo Nome & $\mathbf{0 , 0 0 8}$ & $0,090 *$ \\
\hline Profissionais de Saúde Gritaram ou Bateram & $0,256^{*}$ & $1,000 *$ \\
\hline Sofreu Violência & $1,000^{*}$ & $0,381^{*}$ \\
\hline
\end{tabular}

*Teste de Fischer (menor frequência esperada $<5$ )

† Procedimento exequível apenas no momento do parto

A presença do acompanhante durante o trabalho de parto e parto esteve associada à maior realização de tecnologias do cuidado como massagens e oferta de banhos, assim como ao uso de técnicas de respiração durante o momento do parto $(0,016)$. As mulheres acompanhadas deambularam mais e tiveram maior liberdade de mudanças de posição durante o processo parturitivo.

No rol das intervenções obstétricas, houve importante associação na realização da Manobra de Kristeller $(0,016)$ e na administração endovenosa de ocitocina durante o trabalho de parto $(0,009)$, sendo ambas utilizadas com menor frequência quando em presença do acompanhante.

O acompanhamento foi determinante ainda na forma como os profissionais de saúde se relacionaram com a parturiente, evidenciando-se preocupação da equipe em se identificar, dirigir-se à mulher pelo nome e fornecer orientações quanto aos procedimentos a serem realizados, como os motivos da infusão de ocitocina. O déficit na educação em saúde emergiu nas questões relativas às informações gerais sobre a evolução do trabalho de parto, mas este não foi atrelado à existência do acompanhante, estando relacionado ao conhecimento dos profissionais de saúde sobre o processo parturitivo em si.

Os benefícios também foram evidenciados na experiência parturitiva das mulheres a partir dos sentimentos expressos pelas mesmas com menores percentuais na percepção de abandono ou sensação de isolamento, seguido da inferior vivência de desrespeito durante o parto $(0,027)$.

\section{DISCUSSÃO}


O perfil assistencial e os dados obstétricos observados na cidade de Niterói-RJ, vão ao encontro do perfil obstétrico nacional, observando-se inúmeras semelhanças. Atualmente, $55 \%$ das gestantes brasileiras não desejaram a gravidez atual, confirmando ainda as expectativas epidemiológicas de mudança no perfil obstétrico com queda crescente nas taxas de natalidade.

Quanto ao perfil sociodemográfico, os critérios que versam sobre etnia, faixa etária e classe econômica divergem do padrão nacional atual, no qual as mulheres foram caracterizadas como predominantemente pardas, com faixa etária de 20 a 24 anos, pertencentes à classe $\mathrm{C}$, a partir dos critérios da Associação Brasileira de Empresas de Pesquisas (ABIPEME) ${ }^{(16)}$.

Em linhas gerais, o acompanhante mostrou-se fortemente presente no momento da internação, sugerindo que as gestantes chegam acompanhadas na Maternidade. Entretanto, progressivamente, o acompanhante de livre escolha da mulher vai se afastando ao longo das etapas do processo. Mesmo que não mensurados o contexto e os motivos da ausência dos acompanhantes nos cenários elencados, sugerem-se fortes relações com a adoção de rotinas hospitalares nas Unidades de Saúde, que não favorecem o acompanhamento por pessoa de livre escolha da mulher durante o processo parturitivo, contrariando a Legislação vigente ${ }^{(5)}$.

Quando em pauta a implementação da presença de acompanhantes durante a internação para o parto, 56,7\% das mulheres que pariram em território nacional tiveram um acompanhante em algum dos momentos, caracterizando predominância da ausência parcial. Há ausência total de acompanhantes em $24,5 \%$ dos casos, e não mais que $18,8 \%$ das mulheres desfrutaram de sua presença 42,1\% contínua. Quando analisados isoladamente os momentos do acompanhamento durante a internação para o parto, $70,1 \%$ das parturientes estiveram acompanhadas antes/durante a admissão, durante o trabalho de parto, $32,7 \%$ no momento do parto e nascimento, e $61,3 \%$ no pós-parto ${ }^{(6)}$.

No tocante à prevalência do acompanhante durante o puerpério nas maternidades públicas de Niterói-RJ, houve grande divergência entre os estudos, uma vez que o acompanhante esteve presente em, aproximadamente, metade do quantitativo de mulheres observadas em nível nacional. Contudo, embora a permanência do acompanhante de livre escolha da mulher ainda deva ser objeto de discussões visando ampliar sua inserção no ambiente hospitalar obstétrico, os índices encontrados revelaram maior permanência do acompanhante durante a maioria das fases de internação, em relação aos dados disponíveis nacionalmente ${ }^{(6)}$.

Neste estudo, a ocorrência de Manobra de Kristeller esteve intimamente relacionada à ausência do acompanhante no momento do parto e nascimento, sendo realizada em menor proporção nas mulheres acompanhadas, sugerindo maior empoderamento dessas e tornando questionáveis as bases que norteiam o profissional para a indicação do procedimento.

Igualmente, a ocitocina endovenosa foi menos prescrita durante o trabalho de parto para as mulheres acompanhadas, indo ao encontro dos achados da literatura, que comprovam o estímulo aos níveis de ocitocina endógena provocado pela maior sensação de segurança e relaxamento, evocado a partir do acompanhamento intraparto por pessoa de livre escolha. Os efeitos deste hormônio foram comprovados a partir da observação de menor uso de analgesia intraparto, assim como do menor tempo de trabalho de parto vivenciado por tais mulheres ${ }^{(16)}$.

Sabe-se que a administração endovenosa de ocitocina, a realização de amniotomia e da Manobra de Kristeller, são intervenções importantes que podem agregar riscos e, por isso, somente devem ser realizadas baseadas em indicações precisas, devendo o seu uso rotineiro ser definitivamente abandonado ${ }^{(17)}$.

Neste estudo, a presença do acompanhante de livre escolha da mulher teve o potencial de aumentar a ocorrência de massagens, os banhos terapêuticos, a utilização de técnicas de respiração, a liberdade de deambulação e a movimentação. Foram também descritas maiores taxas de parto vaginal espontâneo com menores intervenções, sendo o acompanhamento mais eficaz quando realizado por pessoa não integrante da equipe hospitalar ou da rede social da mulher. Entretanto, sugere-se que, aparentemente, não há impacto em outras intervenções realizadas durante o processo de parturição ${ }^{(16)}$.

A presença do acompanhante teve forte influência na qualidade da vivência do processo parturitivo, sendo determinante na redução da percepção das situações de abandono, isolamento e desrespeito, 
todas compreendidas nos conceitos de violência obstétrica. Recentemente, constatou-se que uma em cada quatro mulheres brasileiras relatou ter experienciado situações de violência obstétrica e desrespeito durante o trabalho de parto e parto, com frequência de $74 \%$ em maternidades na rede do SUS ${ }^{(18-19)}$.

Especificamente sobre a atitude dos profissionais, a presença do acompanhante no processo parturitivo foi associada ao tratamento diferenciado, mais individualizado, com maior recebimento de orientações e esclarecimentos acerca dos procedimentos a serem realizados. Assim como na Experiência de Hawthorne, quando se observou que as respostas e comportamentos dos indivíduos são modulados quando estão sob observação, fica explícita a influência positiva da presença do acompanhante no cuidado instituído pelos profissionais de saúde ${ }^{(20)}$.

Concluindo, o suporte contínuo durante o processo parturitivo possui relevantes benefícios em todos os âmbitos para as mulheres e os bebês, sem risco conhecido, favorecendo a condução fisiológica do processo. Assim sendo, todas as mulheres deveriam desfrutar do acompanhamento contínuo por pessoa de sua escolha em todas as etapas do processo de parto e nascimento ${ }^{(16)}$.

\section{CONSIDERAÇÕES FINAIS}

Frente ao exposto, conclui-se que há déficit da presença do acompanhante de livre escolha da mulher durante as diversas fases do processo parturitivo nas Maternidades Públicas de Niterói, Rio de Janeiro. Embora avanços se façam presentes, a atenção obstétrica ainda está pautada no modelo de assistência tecnocrático, assim ignorando as recomendações das evidências científicas atuais. Associando o acompanhamento por pessoa de escolha da mulher com os cuidados recebidos, este se mostrou extremamente profícuo com inúmeras implicações em favor da oferta de uma assistência obstétrica diferenciada e de qualidade, sem presunção de malefícios.

Não foi possível desvelar o contexto e os motivos da ausência dos acompanhantes nos diversos momentos do trabalho de parto e parto nos cenários elencados, a partir dos dados analisados, sendo esta uma limitação do presente estudo. Sugere-se a reestruturação das normas, rotinas e estruturas hospitalares com vistas a propiciar a inclusão do acompanhante, de forma a garantir o direito das mulheres usuárias e permitir que todas possam desfrutar de seus benefícios.

\section{REFERÊNCIAS}

1. Ministério da Saúde (BR). Cadernos Humaniza SUS. Humanização do parto e do nascimento. Brasília: Ministério da Saúde;2014.

2. Brüggemann OM, Ebsen ES, de Oliveira ME, Gorayeb MK, Ebele RR. Reasons which lead the health services not to allow the presence of the birth companion: nurses' discourses. Texto Contexto Enferm. [Internet] 2014;23(2) [acesso em 12 ago 2016]. Disponível:http://dx.doi.org/10.1590/0104-07072014002860013.

3. Santos LM, Pereira SSC. Vivências de mulheres sobre a assistência recebida no processo parturitivo. Physis. [Internet] 2012;22(1) [acesso em 20 jun2016]. Disponível: http://dx.doi.org/10.1590/S0103-73312012000100005.

4. Congresso Nacional (BR). Lei n. 11.108, de 07 de abril de 2005. Altera a Lei ${ }^{\circ}$ 8.080, de 19 de setembro de 1990, para garantir às parturientes o direito à presença de acompanhante durante o trabalho de parto, parto e pósparto imediato, no âmbito do Sistema Único de Saúde. Diário Oficial da Republica Federativa do Brasil, Brasília, 7 de abr. 2005. Seção1:1.

5. Ministério da Saúde (BR). Pesquisa Rede Cegonha: Ouvidoria Geral do SUS. Brasília: Ministério da Saúde;2012.

6. Diniz CSG, d'Orsi E, Domingues RMSM, Torres JA, Dias MAB, Schneck CA, et al. Implementação da presença de acompanhantes durante a internação para o parto: dados da pesquisa nascer no Brasil. Cad. Saúde Pública. [Internet] 2014;30(S140-S153) [acesso em 12 jul 2016]. Disponível: http://www.scielo.br/pdf/csp/v30s1/0102-311Xcsp-30-s1- 0140.pdf.

7. Tomeleri KR, Pieri FM, Violin MR, Serafim D, Marcon SS. Eu vi meu filho nascer: vivência dos pais na sala de 
parto. Rev. Gaúcha Enferm. [Internet] 2007;28(4) [acesso em 15 abr 2016]. Disponível: http://www.seer.ufrgs.br/ RevistaGauchadeEnfermagem/article/view/3110.

8. Gonzalez AD, Fernandes ES, da Silva EF, Rabelo M, Souza SRRK. A percepção do acompanhante no processo do nascimento. Cogitare Enferm. [Internet] 2012;17(2) [acesso em 15 abr 2016]. Disponível:http://dx.doi.org/10.5380/ ce.v17i2.27889.

9. Sanches ICP, Couto IRR, Abraão AL, Andrade M. Acompanhamento hospitalar: direito ou concessão ao usuário hospitalizado?. Ciênc. saúde coletiva. [Internet] 2013;18(1) [acesso em 15 abr 2016]. Disponível: http://dx.doi. org/10.1590/S1413- 81232013000100008.

10. Longo CSM, Andraus LMS, Barbosa MA. Participação do acompanhante na humanização do parto e sua relação com a equipe de saúde. Rev. Eletr. Enf. [Internet] 2010;12(2) [acesso em 15 abr 2016]. Disponível:http:// dx.doi.org/10.5216/ree.v12i2.5266.

11. Aires NT, Meincke SMK, Corrêa ACL, Alves CN, Fernandes RFM, Palma JS, et al. Aplicabilidade dos direitos das parturientes: do paradigma à realidade. Saúde (Santa Maria). [Internet] 2015;41(1) [acesso em 15 abr 2016]. Disponível: http://dx.doi.org/10.5902/2236583416195.

12. Santos LM. Análise da atenção à saúde da mulher no ciclo gravídico e puerperal. [dissertação]. Salvador (BA): Universidade Federal da Bahia;2010.

13. Rodrigues DP. Violência obstétrica no processo do parto e nascimento da região Metropolitana II do Estado do Rio de Janeiro: percepção de mulheres/puérperas. [dissertação]. Niterói (RJ): Universidade Federal Fluminense;2014.

14. Viera S. Introdução à bioestatística. 5ª ed. Rio de Janeiro: Elsevier;2015.

15. Ministério da Saúde (BR). Conselho Nacional de Saúde. Diretrizes e normas regulamentadoras de pesquisa envolvendo seres humanos. Resolução n. 466, de 12 de dezembro de 2012. Brasília;2012.

16. Leal MC, da Gama SGN. Pesquisa nascer no Brasil: inquérito nacional sobre parto e nascimento. Cad. Saúde Pública. [Internet] 2014;30(Supl.1) [acesso em 12 ago 2016]. Disponível:http://dx.doi.org/10.1590/0102311XED01S114.

17. Hodnett ED, Gates S, Hofmeyr G, Sakala C, Weston J. Continuous support for women during childbirth. Cochrane Database Syst Rev. [Internet] 2011;16(2) [acesso em 20 Jun2016]. Disponível:http://dx.doi. org/10.1002/14651858.CD003766.pub3.

18. Reis LGC, Pepe VLE, Caetano R. Maternidade segura no Brasil: o longo percurso para a efetivação de um direito. Physis. [Internet] 2011;21(3) [acesso em 10 jun 2016]. Disponível: http://dx.doi.org/10.1590/S010373312011000300020 .

19. Fundação Perseu Abramo. Gravidez, filhos e violência institucional no parto. [Internet] 2010 [acesso em 11 jan 2016]. Disponível:http://csbh.fpabramo.org.br/node/7247.

20. Mayo E. The human problems of an industrial civilization. New York: The Macmillan Company;1933. 